\title{
Research Paper \\ Postural Control Status in Relation to Anthropometric and Postural Indices of Active People
}

Parisa Sedaghati ${ }^{1},{ }^{*}$ Hamid Zolghadr $^{1}$, Hasan Daneshmandi $^{1}$

1. Department of Corrective Exercises and Sport Injuries, Faculty of Physical Education and Sport Sciences, University of Guilan, Rasht, Iran.

Citation: Sedaghati P, Zolghadr H, Daneshmandi H. [Study Postural Control in Relation to Anthropometric and Postural Indexes of Active Participants (Persian)]. Journal of Sport Biomechanics. 2019; 5(1):50-61. https://doi.org/10.32598/biomechanics.5.1.5

https://doi.org/10.32598/biomechanics.5.1.5

Key words:

Postural Control,

Balance, Anthropometric, Active students

\section{ABSTRACT}

Objective The purpose of this study was to investigate the postural control and its relation to some anthropometric and balance indices in active male college students.

Methods The study population consisted of all active students (mean age: $21.15 \pm 2.02$ years; mean height:1.78 $\pm 0.07 \mathrm{~cm}$; mean weight: $71.50 \pm 10.15 \mathrm{~kg}$; body mass index: $22.45 \pm 2.53 \mathrm{~kg} / \mathrm{m2}$ ) at University of Guilan in the academic year 2018-2019. Of these, 40 male students in physical education were selected randomly. The anthropometric and balance indices were chest width, chest depth, chest circumference, forward head, round shoulder, kyphosis and lordosis. For postural control assessment, single-leg stance test was used in four different position. Pearson correlation test and linear regression analysis were used to examine the relationship between variables.

Results There was a significant relationship between the forward head and first condition $(r=+0.633)$ and between the chest depth and third condition $(r=-0.409)$, fourth condition $(r=-0.372)$, total condition $(r=$ -0.428), and between the chest circumference and third condition ( $r=-0.354)$. Kyphosis, lordosis, round shoulder, and chest width had no significant relationship with the postural control.

Conclusion There was a significant but weak relationship between postural control and some anthropometric and balance indices. Hence, it seems that anthropometric and balance indicators alone cannot affect the postural control of active and healthy male college students.

\section{Extended Abstract}

\section{Introduction}

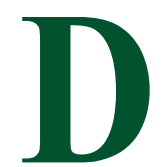

aily exercise and activities require postural control known as the ability to monitor body posture and alignment in space and include multilateral analysis of the musculoskeletal and nervous systems [1]. Sensory inputs are one of the basic requirements for postural control in motion systems, so reducing afferent feedback can disrupt the balance, which can lead to an increase in the risk of injury during exercise [2].

The system of monitoring the body posture in space has always attracted the attention of researchers and a lot of research has been conducted on this topic. Results showed that the visual, somatic, and vestibular sensory messages cause proper balance and maintain proper body alignment [1].

Posture is defined as the combination of the position of the joints of the body concerning each other at the same time.

\section{* Corresponding Author:}

Hamid Zolghadr, Msc.

Address: Department of Corrective Exercises and Sport Injuries, Faculty of Physical Education and Sport Sciences, University of Guilan, Rasht, Iran

Tel: +98 (937) 5636180

E-Mail: 94hamidzolghadr@gmail.com 
The position of each joint will affect the position of the other joints. Proper posture is a situation where the joints and muscle activities are pressed at their lowest. Other situations that put pressure on the joints are commonly referred to as false postures, which consume a lot of energy. Spinal anomalies, rooted from inherited, acquired, and/or unknown causes, affect postural control and disrupt one's balance [3].

\section{Participants and Methods}

The research was causal-comparative and its data were quantitatively measured. The statistical population was male undergraduate students (mean age: $21.15 \pm 2.02$; height: 1.78 \pm 0.07 ; weight: $71.50 \pm 10.15$; BMI: $2.53 \pm 22.45$ ) from the University of Guilan, in the academic year of 2018-2019. The statistical population was selected by random simple sampling from male students of physical education.

Inclusion criteria included no history of defects in the nervous, visual, auditory, and vestibular systems, dizziness, fracture, and/or surgery in the lower extremities and ankle sprains. 40 students were selected as the sample of the study. The selected anthropometric and posture indices included chest width, chest depth, chest circumference, forward head, round shoulder, kyphosis, and lordosis.

Anthropometric indices were measured using a stadiometer, a digital scale, a flexible tape measure, and a depthometer caliper gauge (to measure chest depth). In addition, a $60 \mathrm{~cm}$ flexible ruler, a camera, and the Kinova software were used to measure posture indices, and the Single Leg Stance (SLS) test was used to evaluate the posture control.

Kolmogorov-Smirnov test was used to determine the normal distribution of data. Correlation and regression tests were used for data analysis by SPSS v. 21 software.

\section{Results}

Table 1 shows the correlations between the mean values of anthropometric indices of posture and postural control of active individuals. Regarding the reported correlations among the predictor, variables with the regression model balance were investigated. Summary results of linear regression test between the criteria variables (balance) and predictor variables (chest depth, head forward, roun $\mathrm{d}$ shoulder) showed that this model was able to predict $22 \%$ variance of criterion variable (postural control) with a correlation coefficient of 0.532 and the coefficient of determination 0.283 (Adjusted R Square=0.223) (Table2).

In Table 3, the results of the regression coefficients test also showed that among the predictor variables, two variables of chest depth and head forward were able to significantly predict balance scores. The results of this relationship indicate that there is a positive relationship between the variables of chest depth and head forward and postural control.

Table 1. Pearson correlation coefficients between anthropometric, postural, and postural control characteristics

\begin{tabular}{|c|c|c|c|c|c|c|}
\hline \multirow{2}{*}{ Variable } & Variables & \multirow{2}{*}{ First Status } & \multirow{2}{*}{ Second Status } & \multirow{2}{*}{ Third Status } & \multirow{2}{*}{ Fourth Status } & \multirow{2}{*}{ Sum of Statuses } \\
\hline & Values & & & & & \\
\hline \multirow{2}{*}{ Kyphosis } & $r$ & 0.147 & 048.0- & 0.105 & 0.146 & 0.116 \\
\hline & $P$ & 0.364 & 769.0 & 0.519 & 0.367 & 0.475 \\
\hline \multirow{2}{*}{ Lourdes } & $r$ & 0.197 & $133.0-$ & -0.93 & 0.018 & -0.043 \\
\hline & $\mathrm{P}$ & 0.224 & 414.0 & 0.057 & 0.912 & 0.079 \\
\hline \multirow{2}{*}{ Forward head } & r & $0.433^{*}$ & 101.0 & 0.099 & 0.176 & 0.164 \\
\hline & $\mathrm{P}$ & 0.005 & 536.0 & 0.543 & 0.278 & 0.313 \\
\hline \multirow{2}{*}{ Round shoulder } & r & -0.098 & 060.0- & -0.207 & -0.192 & -0.206 \\
\hline & $P$ & 0.546 & 711.0 & 0.201 & 0.243 & 0.203 \\
\hline \multirow{2}{*}{ Chest depth } & $r$ & 0.297 & $283.0-$ & -0.409 & $0.372^{* *}$ & $-0.428^{*}$ \\
\hline & $\mathrm{P}$ & 0.063 & 077.0 & 0.009 & 0.018 & 0.006 \\
\hline \multirow{2}{*}{ Chest circumference } & r & -0.201 & 196.0- & $-0.354 * *$ & 0.161 & 0.273 \\
\hline & $\mathrm{P}$ & 0.214 & 224.0 & 0.025 & 0.032 & 0.088 \\
\hline \multirow{2}{*}{ Chest width } & $r$ & -0.185 & 005.0 & -0.216 & 0.133 & -0.166 \\
\hline & $\mathrm{P}$ & 0.252 & 975.0 & 0.181 & 0.415 & 0.307 \\
\hline rrelation at the sig & level 0 . & & & & & $\begin{array}{l}\text { surnal of } \\
\text { oort Biomechanics }\end{array}$ \\
\hline
\end{tabular}

** Correlation at the significant level 0.05 
Table 2. Analysis of variance

\begin{tabular}{ccccc}
\hline Source of Changes & The Sum of Squares & Degrees of Freedom & Average of Squares & F-value \\
\hline Regression & 7.463 & 3 & 2.488 & 4.735 \\
Residual & 18.912 & 36 & 0.525 & - \\
Total & 26.375 & 39 & - & - \\
\hline
\end{tabular}

Table 3. Regression model coefficients

\begin{tabular}{cccccc}
\hline Title & Coefficient Estimation & Standard Error & Standardized Coefficient & Test Statistics & $\mathbf{P}$ \\
\hline Fixed & 0.715 & 1.674 & - & 0.427 & 0.672 \\
Chest depth & -0.142 & 0.070 & -0.291 & -2.040 & $0.049^{*}$ \\
Round shoulder & -0.007 & 0.015 & -0.069 & -0.484 & 0.632 \\
Head forward & 0.081 & 0.026 & 0.438 & 3.103 & $0.004^{*}$ \\
\hline & & & & Journal of \\
& & & & Sport Biomechanics
\end{tabular}

Statistical test: linear regression; Predictive variables: chest depth, round shoulder, head forward; Criterion variables: balance

* $\mathrm{P}<0 / 05$

\section{Discussion}

According to the results of this study, there is a weak significant relationship between some anthropometric and postural indices with postural control. Therefore, it seems that anthropometric and postural indices alone cannot affect postural control status of active and healthy male students. Since there is a difference in balance evaluation methods, and anthropometric and postural samples and indices in different studies, it is not possible to sum up a comprehensive conclusion. Also, balance prediction and postural control separately based on factors do not appear to be correct, since balance preservation is the result of the complex interaction of multiple internal and external items and factors such as muscle activity, activated muscle synergy, type of balance strategy, and proprioceptive must also be taken into consideration.

\section{Conclusion}

Based on the results, none of the anthropometric and postural indices used in this study alone can affect postural control in active and healthy subjects. Therefore, further research is needed to find the relationships of the variables involved in postural control and maintaining balance.

\section{Ethical Considerations}

Compliance with ethical guidelines

All ethical principles were considered in this article. The participants were informed about the purpose of the research and its implementation stages; they were also assured about the confidentiality of their information; Moreover, They were allowed to leave the study whenever they wish, and if desired, the results of the research would be available to them.

Funding

This research did not receive any specifc grant from funding agencies in the public, commercial, or not-forproft sectors

\section{Authors' contributions}

Conceptualization, methodology, supervision: All authors; Invesigation, writing original draft, funding acquisition, Resources: Hamid Zolghadr; Writing-review and editing: Parisa Sedaghati, Hassan Daneshmandi.

\section{Conflicts of interest}

The authors declared no conflict of interes.

\section{Acknowledgements}

We would like to thank the research assistant of the University of Guilan and all the subjects who sincerely participated in the present study. 


\title{
بررسى وضعيت كنترل ياسجر در ارتباط با شاخصهاى أنترويومتريكى و وضعيتى افراد فعال
}

\author{
بريسا صداقتى' ، "حميد ذوالقدر' ، حسن دانشمندى'
}

1. كروه آسيبشناسى ورزشى و حركات اصلاحى، داتشكده تربيتبدنى وعلوم ورزشى، دانشكاه كيلان، رشت، ايران.

\begin{abstract}
حكيد

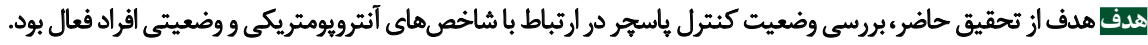

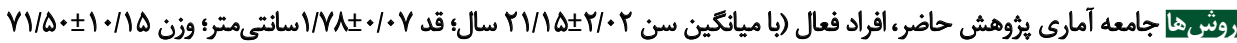

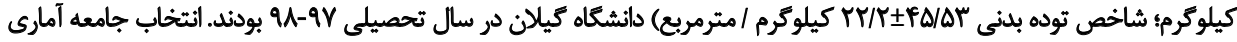

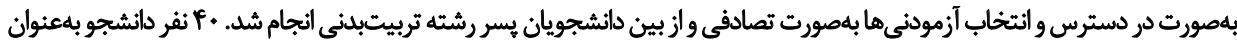

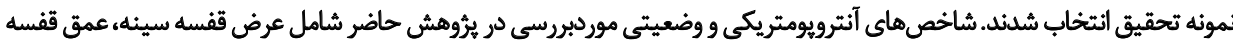

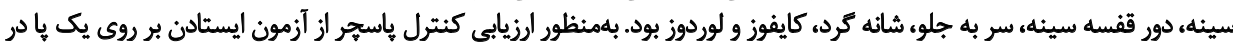

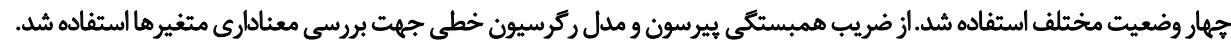

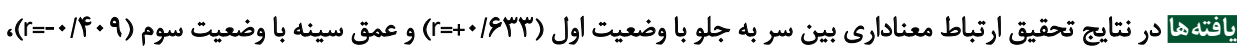

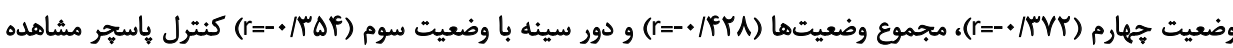

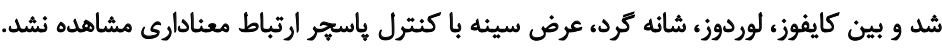

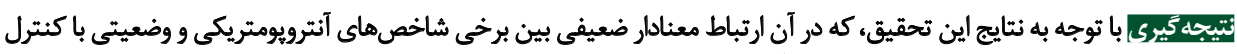

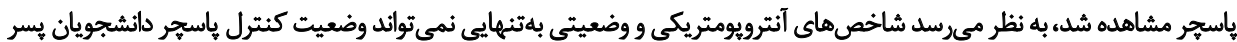
فعال و سالم راتحت تأثير قرار دهد.
\end{abstract}

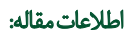

تاريخ دريافت: 19 آذر Vو

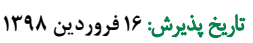

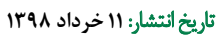

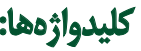

كنترل ياسجر،

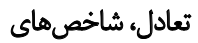

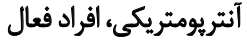

تعريف مى شود. موقعيت هر مفصل بر روى موقعيت ديكر مفاصل

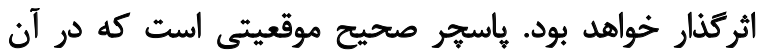

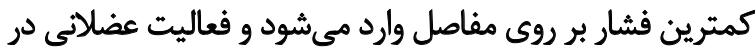

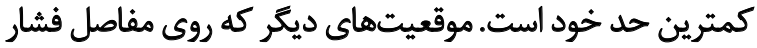

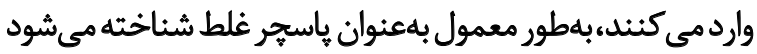

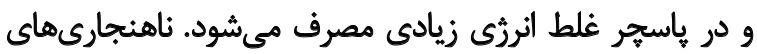

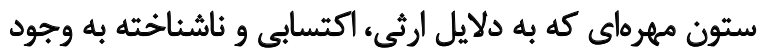

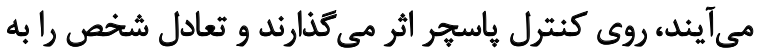

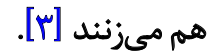

عوامل توناتون نوروفيزيولوريك و مكانيكى مى توانند تعادل راني

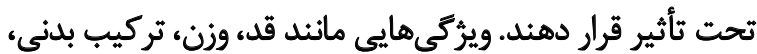

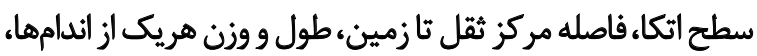

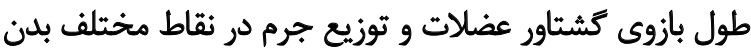

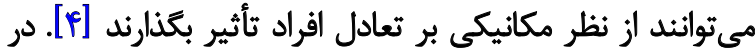

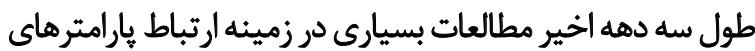

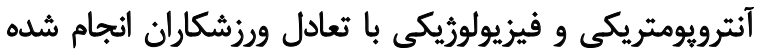

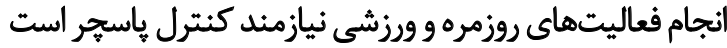

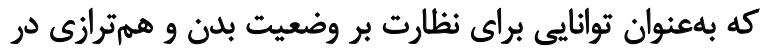

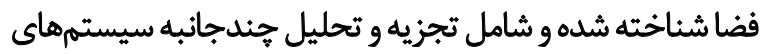

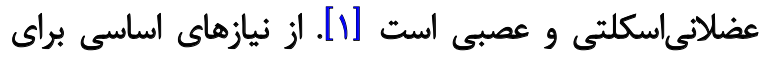

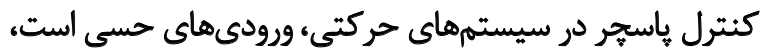

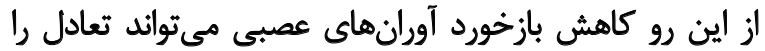

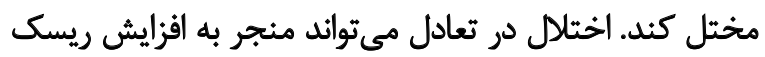

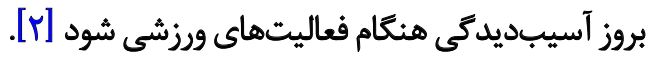
سيستم كنترل وضعيث قراركرفتن بدن در فضا همواره توجه

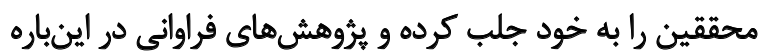

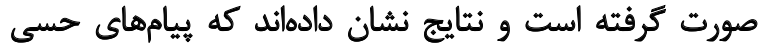

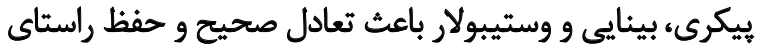

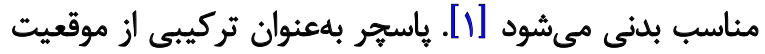

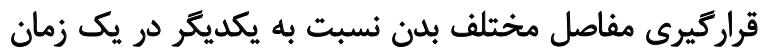




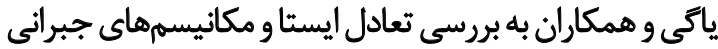

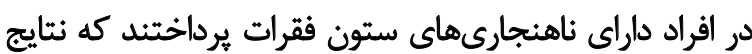

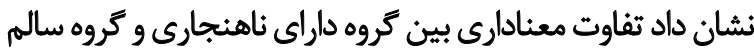

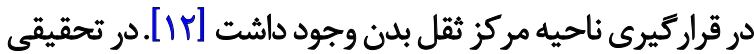

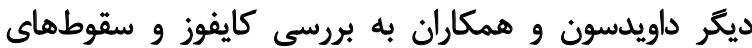

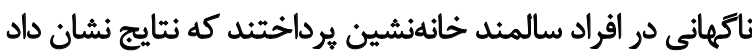

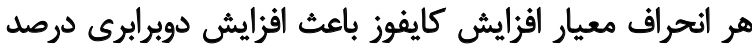

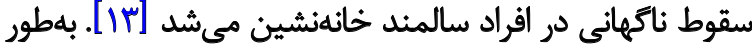

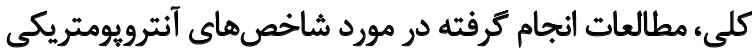

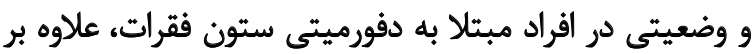

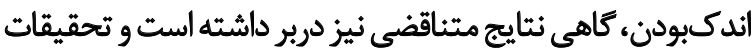

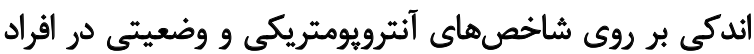
كايفوزيس و لوردوزيس متمركز شدهاند.

با توجه به اينكه ناهنجارىهاى ستون فقرات ممكن است

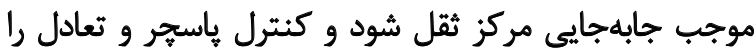

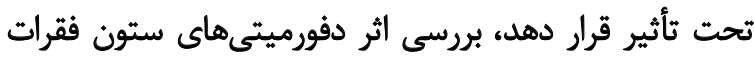

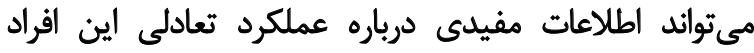

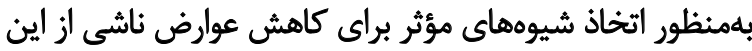

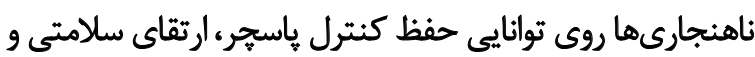

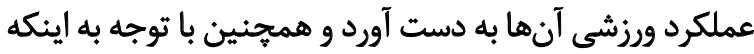

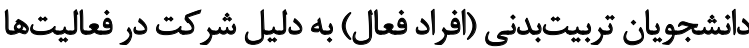

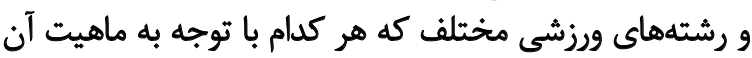

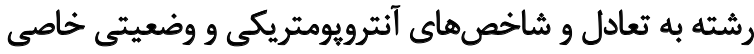

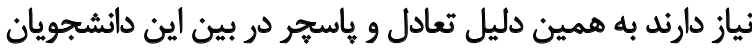

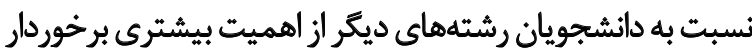

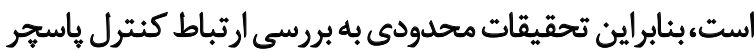

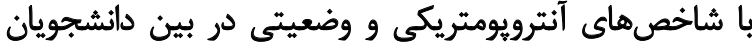

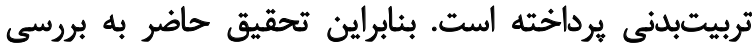

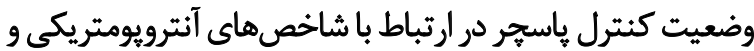
وضعيتى افراد فعال مي يردازد.

$$
\text { روش شُشنى }
$$

تحقيق حاضر از نوع علّى _مقايسهاي بود و دادههاي آن بهصورت

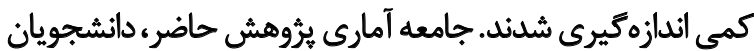

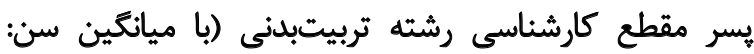

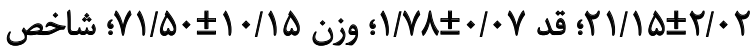

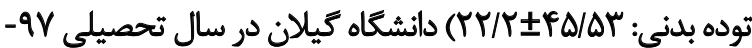

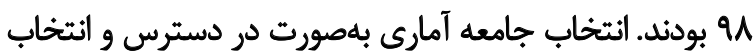

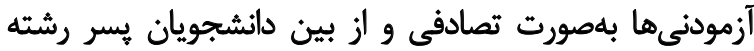

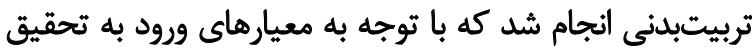

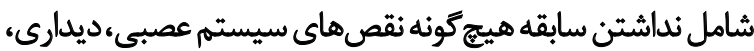

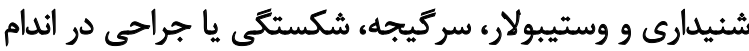

تحتانى و اسيرين مج ويا بود.

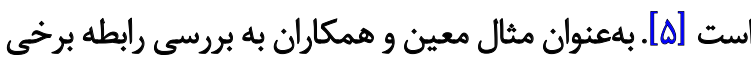

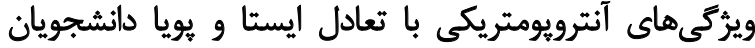

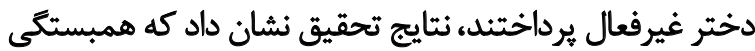

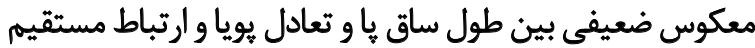

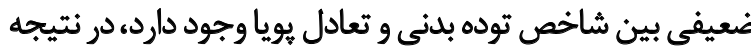

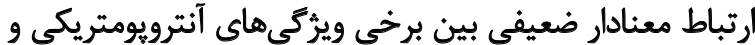

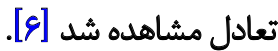

برنجيان و همكاران در تحقيقى به مقايسه تعادل ايستا و

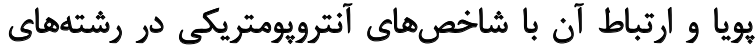

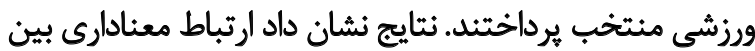

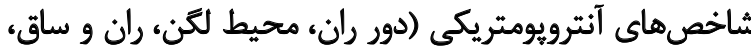

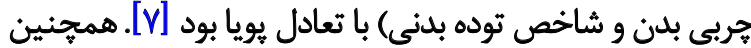

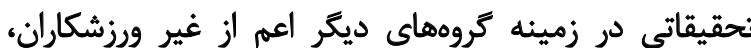

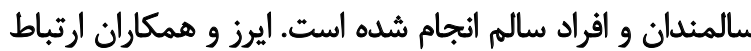

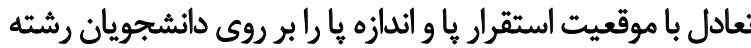

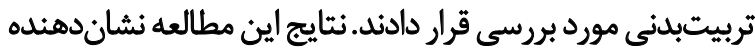

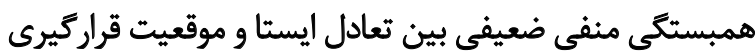

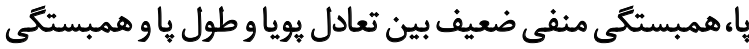
مثبت ضعيفى بين تعادل ايستاو يهناى ياشنه بود [N]] كيم و همكاران در تحقيقى به بررسى رابطه برخى يارارمترهاي

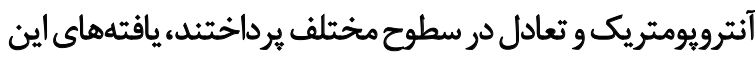

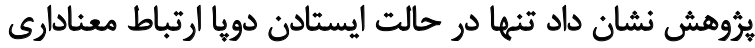

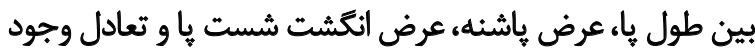

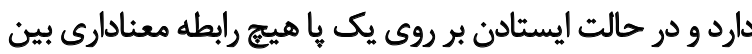

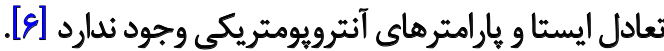

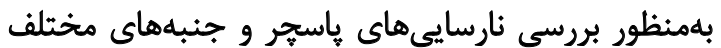

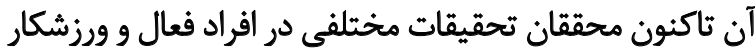

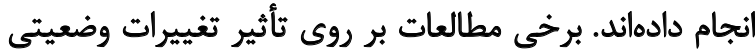

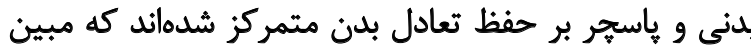

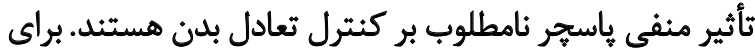

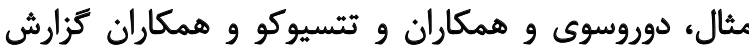

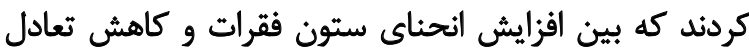

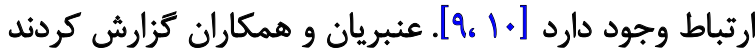

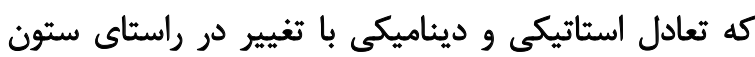

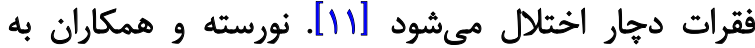
بررسى تعادل در دانشآموزان با قوس اختئ افزايشيافته كايفوز و

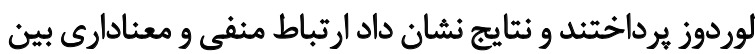

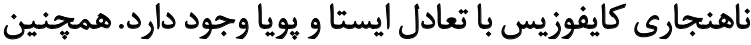

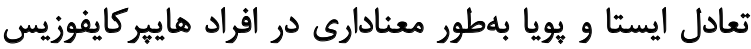

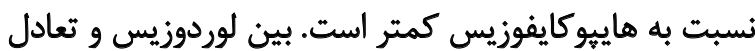

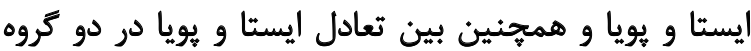

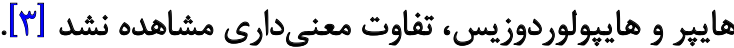




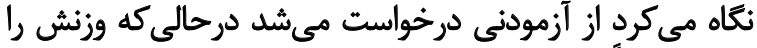

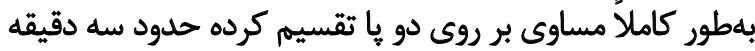

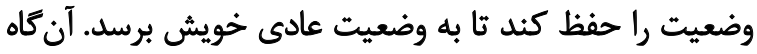

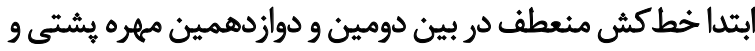

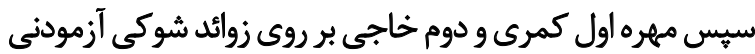

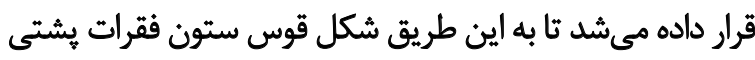

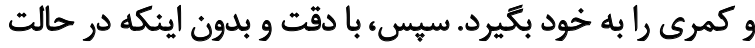

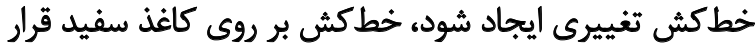

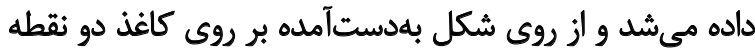

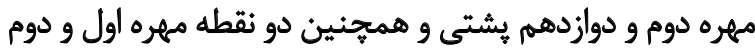

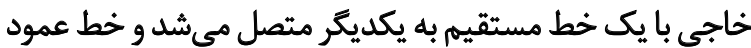

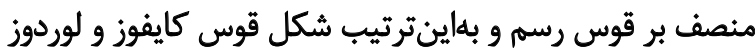

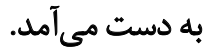
مراحل اندازهيرى و محاسبه زاويه قوس ستون فقرات

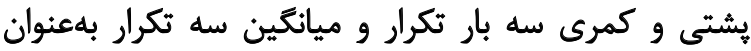

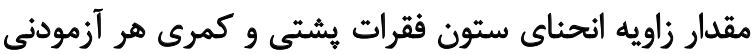
ثبت شد. سيس زاويه كايفوز و لوردوز با استفاده الز فرئ فرمول شماره

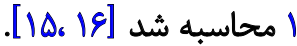

\section{1. $\vartheta=4 \arctan \left(\frac{2 h}{l}\right)$}

براى اندازهيرى زواياى سر و شانه به جلو با استفاده از روش

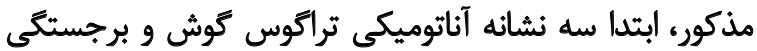

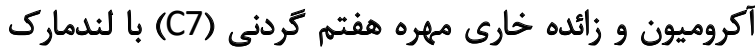

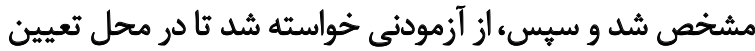

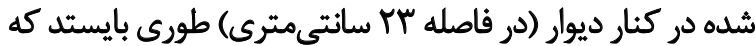

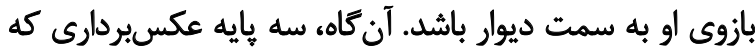

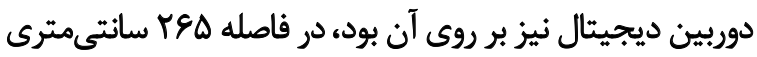

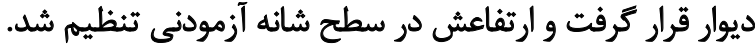

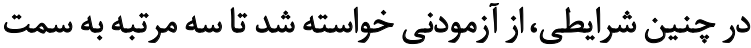

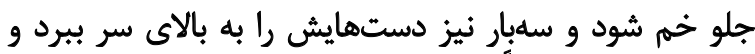

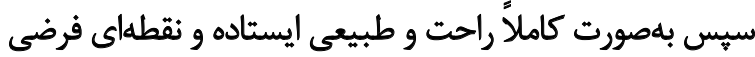

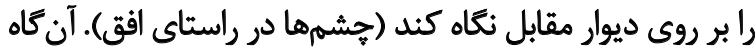

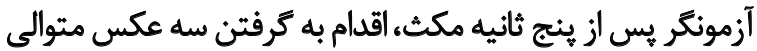

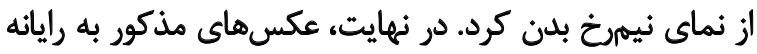

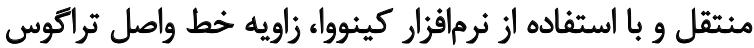

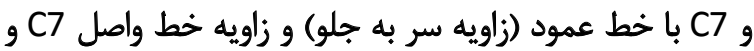

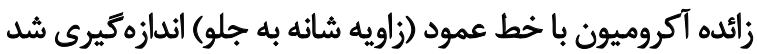

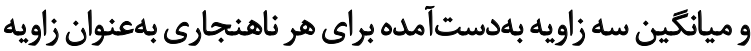

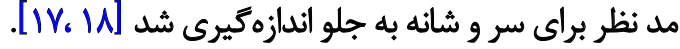
آبمنظور بررسى توانايى افراد در كنترل وضعيت ايستاده، از ازئ

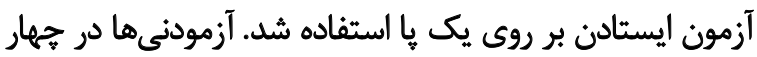

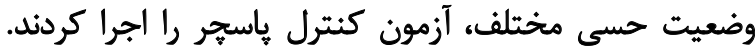

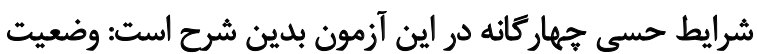

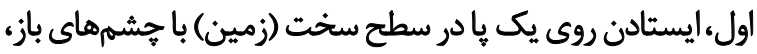

نفر دانشجو بهعنوان نمونه تحقيق انتخاب شدئد. f.

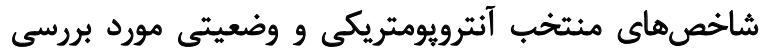

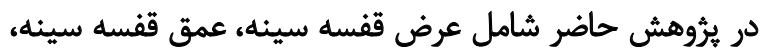

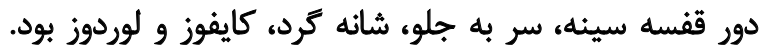

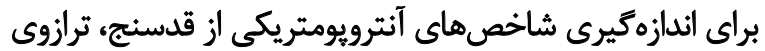

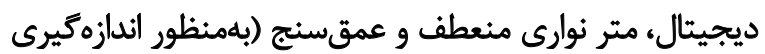

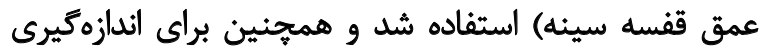
شاخصهاى وضعيتى از خطكش منعطف نو •4 سانتىمترى،

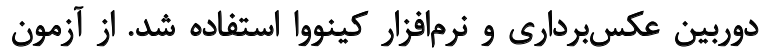

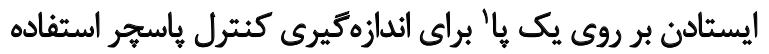

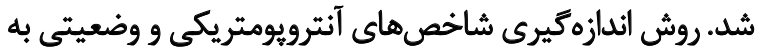

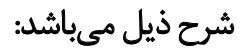

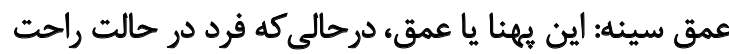

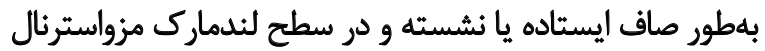

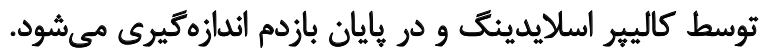

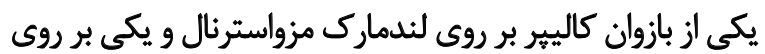

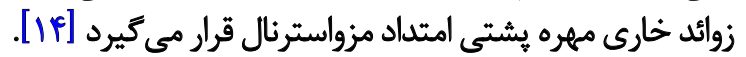
ايهنا (عرض) قفسه سينه: درحالى كه آزمودنى در حالت

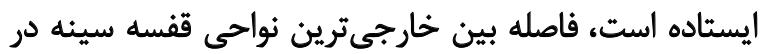

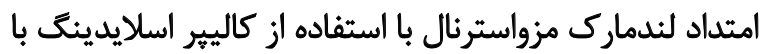

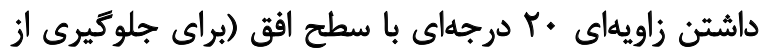

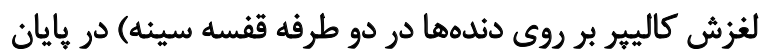

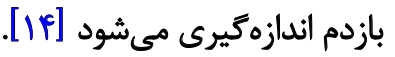

محيط (دور) قفسه سينه: دور قفسه سينه در سطح لندمارك

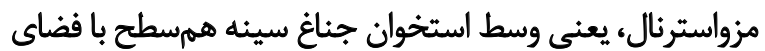

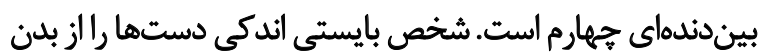

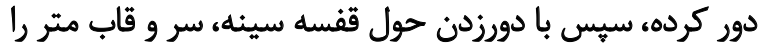

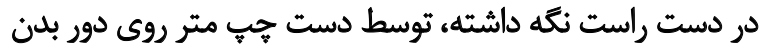

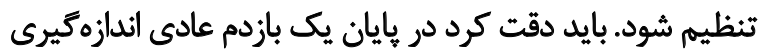

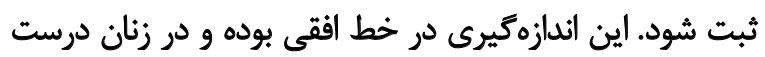

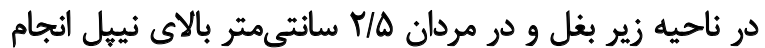

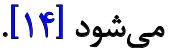

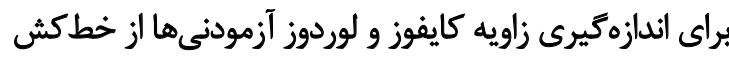

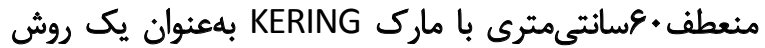

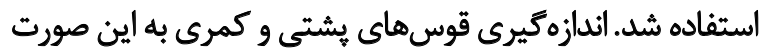

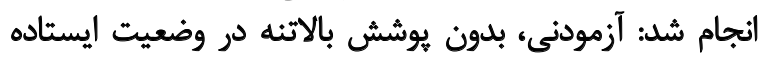

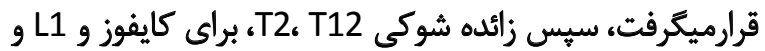

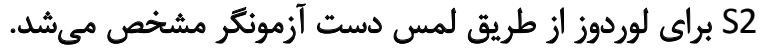

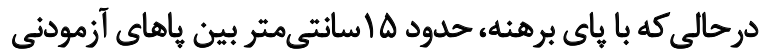

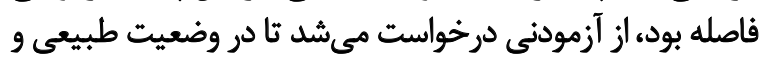
راحت بايستد. سر در حالت طبيعى قرار داشت و آزمودنى بادئ به جلو 
كلموكروف|سميرنوف استفاده شد. براي تجزيه و تحليل دادهها از

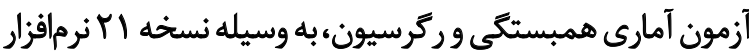

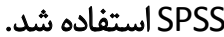

نتايج

طبيعىبودن توزيع دادهها توسط آزمون كلموكروفاسميرنوف

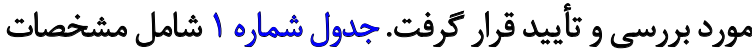
جمعيتشناختى آزمودنى هاست

جلدول شماره Y، شامل همبستكى بين ميانكينهاى شاخصهاى

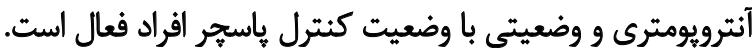

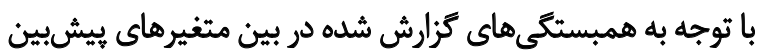

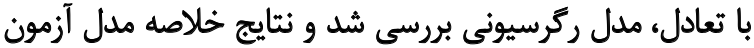

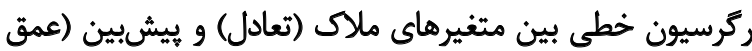

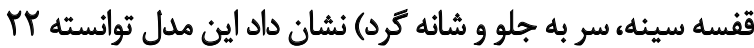

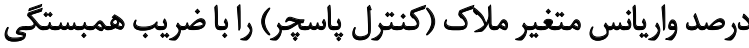

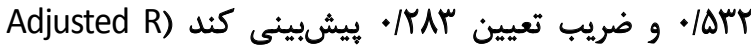

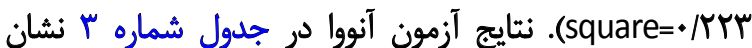

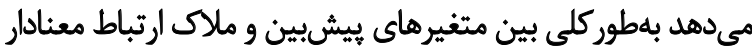

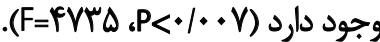

نتايج آزمون ضرايب ركرسيونى در جدول شماره f نيز نشان

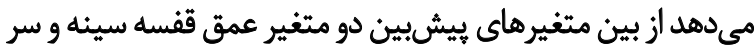

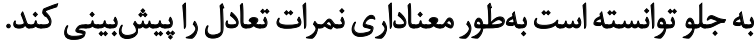

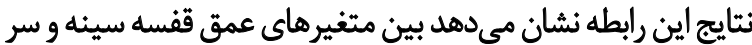

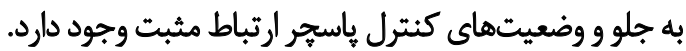

بحث

هدف از انجام اين تحقيق بيشبينى وضعيثهاى كنترل باسجر

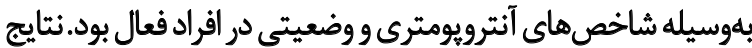
تحقيق نشان داد مدل ركرسيونى در بيشبينى بينى نمرات تعادل توانسته
وضعيت دوم،ايستادن روى يك يا دادر سطح نرم (فوم) باجشمهاي

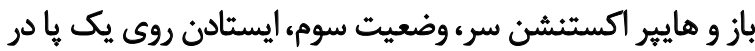

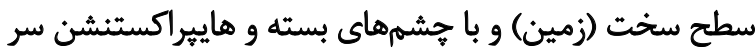

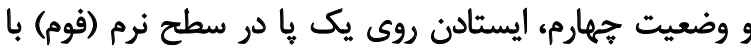

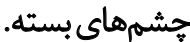

در حالت اول، بهعنوان وضعيت مرجع هيجيَّونه آشفتكي در

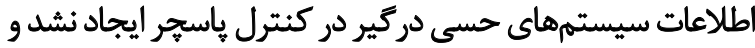

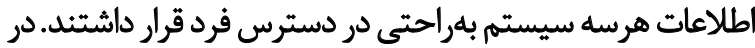

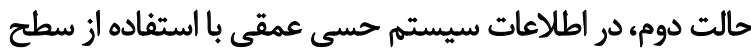

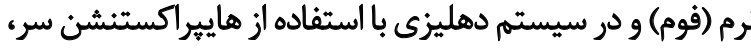

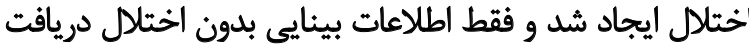

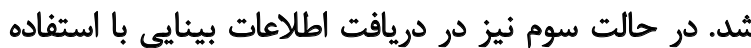

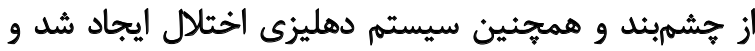

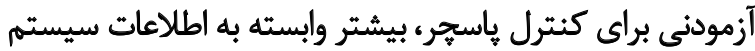

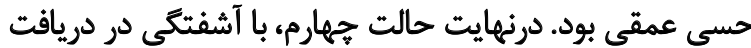

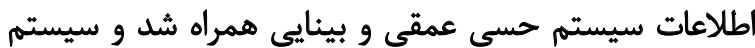

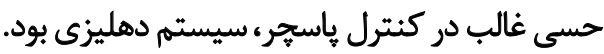

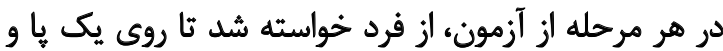

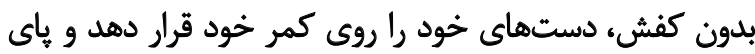

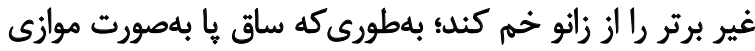

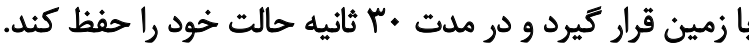

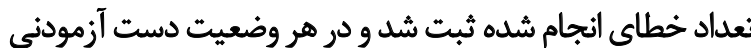

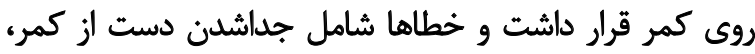

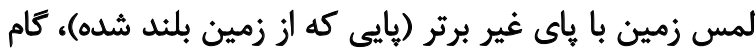

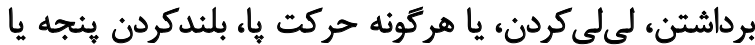

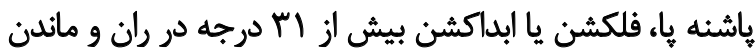

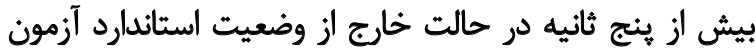

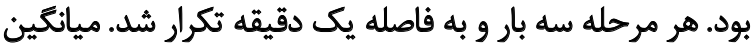

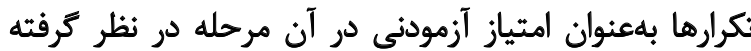

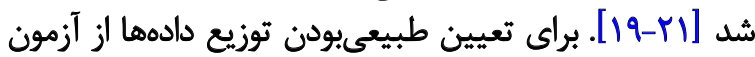

جدول 1. مشخصات جمعيتشناختى آزمودنىها

\begin{tabular}{|c|c|c|c|}
\hline ميانكين \انحراف معيار & شاخصها & ميانكين \$انحراف معيار & شاخصها \\
\hline$Q Y / Y \& \pm \& / \Delta$. & دور قفسه سينه & $T M / \Lambda \Delta \pm Y / \Delta T$ & سن (سال) \\
\hline RV/RTEY/A. & عرض قفسه سينه & $1 / V \wedge \pm . / \cdot V$ & قد (متر) \\
\hline $19 / 91 \pm 1 / 81$ & عمق قفسه سيئه (سائتيمتر) & $V / \Delta \cdot \pm 1 \cdot / 1 \Delta$ & جرم (كيلوكرم) \\
\hline$. / 8 Y \pm \cdot / A r$ & وضعيت اول & $r r / P \Delta \pm Y / \Delta r$ & شاخص توده بدنى (كيلوكرم بر متر مربع) \\
\hline$f / \& \Delta \pm f / \Delta \Delta$ & وضعيت دوم & rE/TAEY/RT & زاويه سر به جلو (درجه) \\
\hline$M T / M T \pm 1 \cdot / 9 r$ & وضعيت سوم & rNVTIV/AT & شانه كرد (درجه) \\
\hline$r q / F q \pm 1 r / v \varepsilon$ & وضعيت جهارم & $r \varphi / N \equiv \pm \Delta / \Delta r$ & لوردوز (درجه) \\
\hline$q V / q r \pm r \Delta / \cdot q$ & مجموع وضعيتها & $M e / e T \pm \& / \pi$ & كايفوز (درجه) \\
\hline
\end{tabular}

مجله بيومكانيك ورنث 
جدول r. ضرايب همبستكى ييرسون بين ويزّكىهاي آنترويومتريكى، وضعيتى وكنترل ياسجر

\begin{tabular}{|c|c|c|c|c|c|c|}
\hline مجموع وضعيتها & وضعيت جهمارم & وضعيت سوم & وضعيت دوم & وضعيت اول & مقادير & متغير \\
\hline.$/ 18$ & / IPS & $+/ 1 \cdot \Delta$ & $-.1+4 \lambda$ & . IFY & $r$ & \multirow{2}{*}{ كايفوز } \\
\hline . / FVA &.$/$ rev & .1019 & . Neq & . WEF & $p$ & \\
\hline$-1 \cdot 1+4$ & $.1 \cdot 11$ & -.1 .94 & " &.$/ 194$ & $r$ & \multirow{2}{*}{ لوردوز } \\
\hline$+/ v 9$ &.$/ 914$ & $\cdot / \Delta V$ &.$/ F I f$ & ./TMY & $p$ & \\
\hline . MAP &.$/ I V E$ & +1.99 & $+/ 1 \cdot 1$ & . & $r$ & \multirow{2}{*}{ سر به جلو } \\
\hline שוM/ &.$/ T V A$ & / & ع & 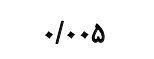 & $p$ & \\
\hline$-+/ r \cdot 9$ &.$- / 194$ & $-\cdot / r+Y$ & -.1 .8 & -1.94 & $r$ & \multirow{2}{*}{ شائه كرد } \\
\hline$\cdot \pi \cdot r$ &.$/ \pi m$ &.$/ r \cdot 1$ & $\cdot M I$ & . lope & $p$ & \\
\hline.$- /$ RTh"* & $-\cdot / \pi r^{*}$ & $-\cdot / f+q \cdots *$ &.$- /$ KAT &.$- /$ Tq & $r$ & \multirow{2}{*}{ عمق سينه } \\
\hline $.1 . .8$ & $.1 \cdot 11$ & .1 .09 & $.1 \cdot w$ & .1 .84 & $p$ & \\
\hline.$/ r n$ &. $\mid 181$ & $-\cdot / \Gamma \Delta P *$ &.$- / 1 q$ & $-\cdot / r+1$ & $r$ & \multirow{2}{*}{ دور سينه } \\
\hline$\% M$ & . & . I. ra &.$/ \pi r \varphi$ &.$/$ IIf & $p$ & \\
\hline$-+/ 98$ & תוז//. &.$- / T I S$ & $1+\infty$ & $-* /$ NA & $r$ & \multirow{2}{*}{ عرض سينه } \\
\hline$\cdot \pi \cdot v$ &.$/ 4 I A$ & +||$\Lambda \mid$ & ./9Yo & . /rat & $p$ & \\
\hline
\end{tabular}

مجله بيومكانيك ورنش

P<0/05 همبستكى در سطح معنادارى"

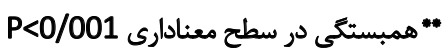

بين كايفوز و كنترل ياسجر وجود نداشت؛ از اين رو نتايج ناهمسو

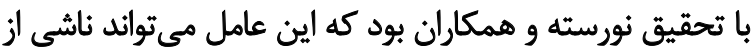

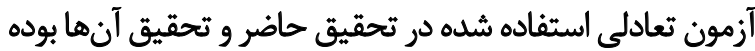

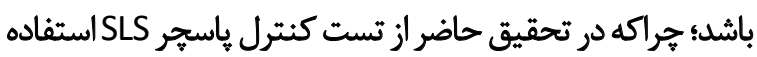

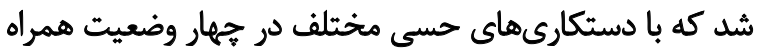

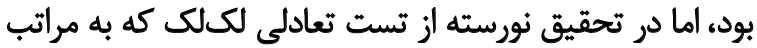

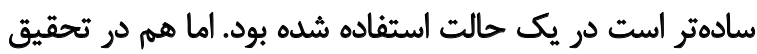

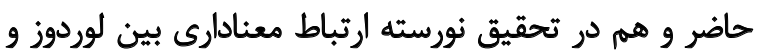

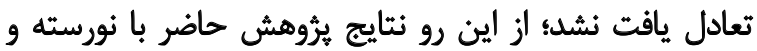

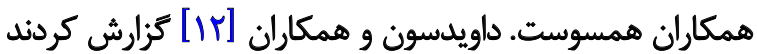

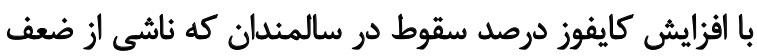

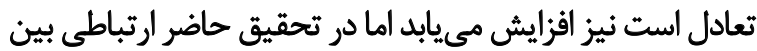

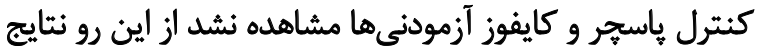

است Tr درصد واريانس كنترل هاسجر را هيشبيني كند. از بين

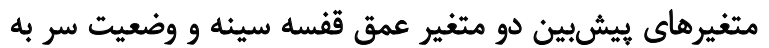

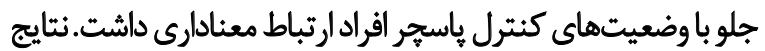

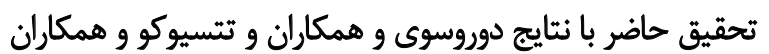

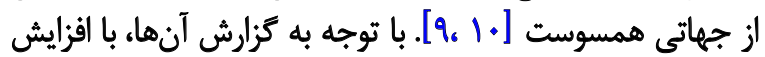

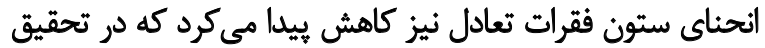

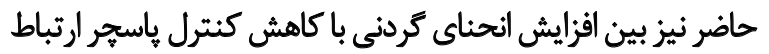

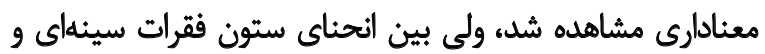

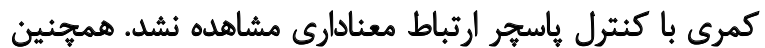

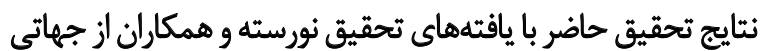

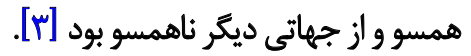
نورسته و همكاران تفاوت معنادارى را بين كايفوز و تعادل مشاهده كردند؛ در صورتى كه در تحقيق حاضر ارتباط معنادارى معن

جدول r. مقادير تحليل واريانس در متغيرهاي يُّوهش

\begin{tabular}{|c|c|c|c|c|c|}
\hline$P$ & $F$ & ميانكين مربعات & درجه آزادى & جمع مربعات & مثبع تغييرات \\
\hline$\cdot 1 \cdot \cdot \gamma^{*}$ & $r / M^{\top} \Delta$ & $T / F M$ & $r$ & V/Fer & ركرسيون \\
\hline- & - & - /Ara & g & WaIr & باقى مانده \\
\hline- & - & - & rq & re/rVD & كل \\
\hline
\end{tabular}


جدول F. ضرايب مدل ركرسيون براى متغيرهاي يُروهش

\begin{tabular}{|c|c|c|c|c|c|}
\hline $\mathbf{P}$ & آماره آزمون & ضريب استانداردشده & خطاى معيار & برأورد ضريب & عنوان \\
\hline.$/ 9 V T$ & ARTV & - & yent & .MIQ & ثابت \\
\hline$+1+4 q^{*}$ & $-r / \cdot r$. &.$- / 491$ &.$/ \cdot v$ &.$- / N F T$ & عمق قفسه سينه \\
\hline $.18 \pi T$ &.$- / F A f$ & -.1 .89 & .1 .10 & $-\bullet / \cdot \bullet V$ & شاثه كرد \\
\hline $.1 .+e^{e *}$ & $r / \mu . r$ & גזA/ & .1 .48 & $\cdot|\cdot 1|$ & سر به جلو \\
\hline
\end{tabular}

مجله بيومكانيك وزنش

آزمون آمارى: ركرسيون خطى، متغيرهاي بيشبين: عمق قفسه سينه، شائه كرد، سر به جلو، متغير ملاك: تعادل

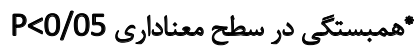

تعادل ايستاو شاخصهاي آنترويومترى ديده نشد كه در تحقيق

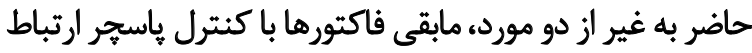

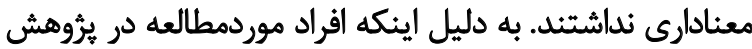

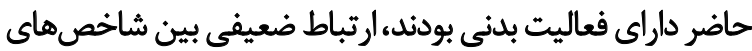

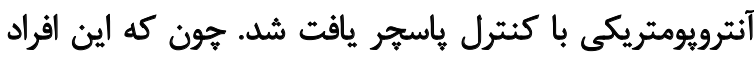

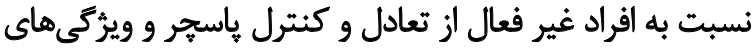

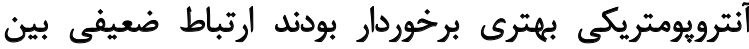

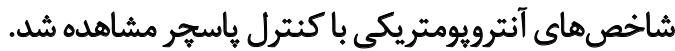

\section{نتيجلكيرى نهايى}

به علت وجود تفاوت در روشهاى ارزيابى تعادل، نمونهها و وخئ

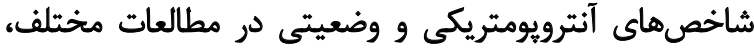

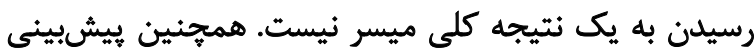

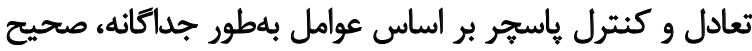

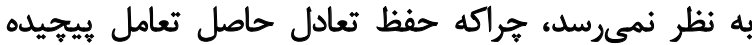

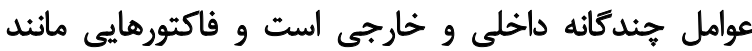

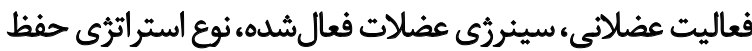

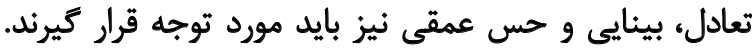

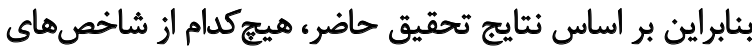

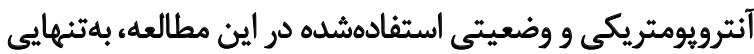

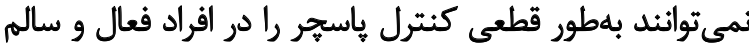

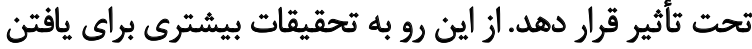

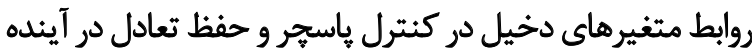

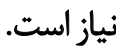

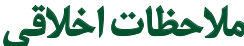

$$
\text { يبيروى از اصول اخلاق بثوهش }
$$

تمامى آزمودنىها به طور داوطلبانه و با تكميل رضايتنامه شخصى در تحقيق حاضر شركت داشتند.

$$
\text { حامي مالى }
$$

اين تحقيق هيج كمك مالى خاصى از سازمانهاى عمومى يا

$$
\text { خصوصى دريافت نكرده است. }
$$

تحقيق حاضر با داويدسون ناهمسوست كه ميتواند به دليل متفاوتبودن نمونهها در هر دو تحقيق باشدا ناهمبات

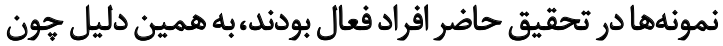

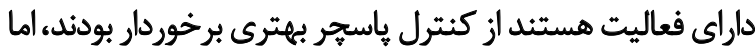

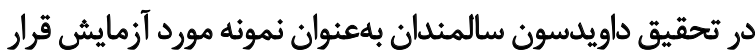

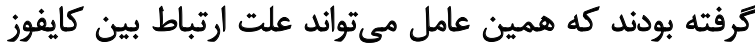

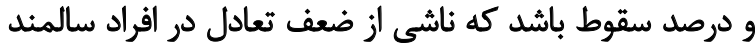

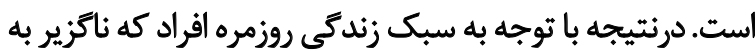

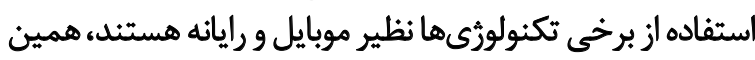

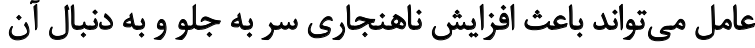

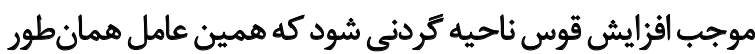

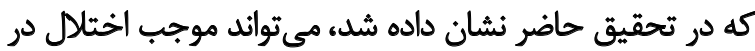

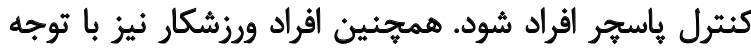

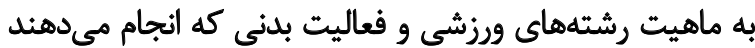

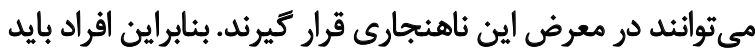

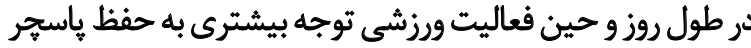

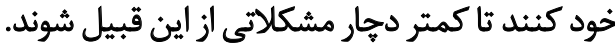

در تحقيق حاضر بين شاخص آنترويومتريكى عمق سينه و

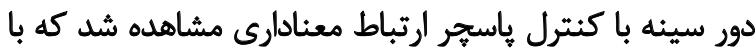

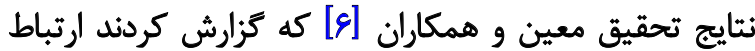

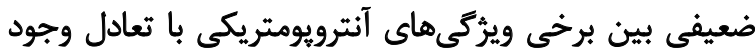

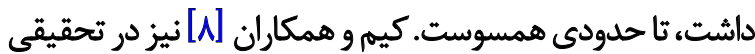

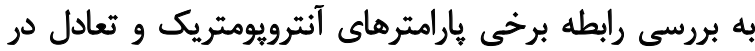

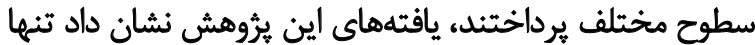

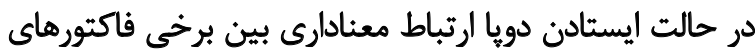

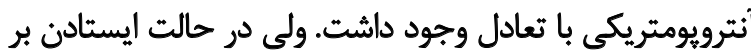
روى يك با هيج رابطه معنادارى بين تعادل ايستا و يارامترهاي آنتاي

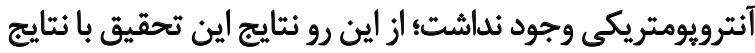

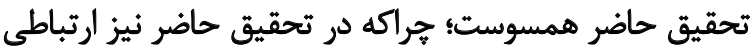

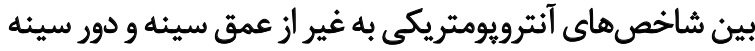

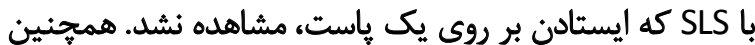

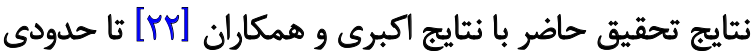
نيز همسوست، خراكه در تحقيق آكبرى ارتباط معنائ معنادارى بين 


$$
\text { مشاركث نويسندكّان }
$$

مفهومسازى،روش شناسى ونظارت: تمامى نويسندكان؛ بررسى

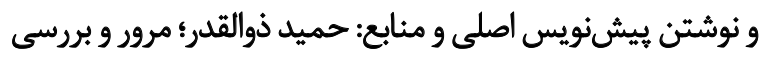
و ويرايش يريسا صداقتى و حسن دانشمندى.

$$
\text { تعارض مثاقع }
$$

بنابر اظهار نويسندكان اين مقاله تعارض منافع ندارد.

$$
\text { تشكر و قدردانى }
$$

بدينوسيله از معاونت يزوهشى دانشكاه تيلان و تمامى تاري

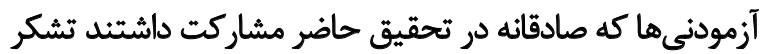

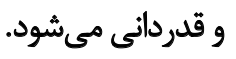




\section{References}

[1] Shumway-Cook A, Woollacott MH. Motor control: Translating research into clinical practice. Philadelphia: Lippincott Williams \& Wilkins; 2007.

[2] Wikstrom EA, Powers ME, Tillman MD. Dynamic stabilization time after isokinetic and functional fatigue. Journal of Athletic Training. 2004; 39(3):247-53. [PMCID] [PMID]

[3] Norasteh AA, Hosseini R, Daneshmandi H, Shah Heidari S. [Balance assessment in students with hyperkyphosis and hyperlordosis (Persian)]. Journal of Sport Medicine. 2014; 6(1):57-71. [DOI:10.22059/JSMED.2014.50131

[4] Palmieri RM, Ingersoll CD, Cordova ML, Kinzey SJ, Stone MB, Krause $B A$. The effect of a simulated knee joint effusion on postural control in healthy subjects. Archives of Physical Medicine and Rehabilitation. 2003; 84(7):1076-9. [DOI:10.1016/S0003-9993(03)00129-1]

[5] Hobbs ML. Dynamic balance and basketball playing ability [MSc. thesis]. San Marcos, Texas: Texas State University; 2008.

[6] Moein E, Movaseghi F. Relationship between some anthropometric indices with dynamic and static balance in sedentery female college students. Turkish Journal of Sport and Exercise. 2016; 18(1):45-9. [DOI:10.15314/tjse.65406]

[7] Berenjian Tabrizi $\mathrm{H}$, Abbasi A, Jahadian $\mathrm{H}$. [Comparing the static and dynamic balance and their relationship with the anthropometric characteristics in athletes of selected sports (Persian)]. Sport Sciences Quarterly. 2014; 6(14):33-46.

[8] Irez GB. The relationship with balance, foot posture, and foot size in school of physical education and sports students. Educational Research and Reviews. 2014; 9(16):551-4. [DOI:10.5897/ERR2014.1790]

[9] Sakamitsu T, Urabe Y, Yamamoto T. Relationship of kyphosis with balance and walking ability in the elderly. Journal of Exercise Physiology. 2007; 22(4):489-94. [DOI:10.1589/rika.22.489]

[10] Çınar E, Akkoç Y, Karapolat H, Durusoy R, Keser G. Postural deformities: Potential morbidities to cause balance problems in patients with ankylosing spondylitis. European Journal of Rheumatology. 2016; 3(1):5-9. [DOI:10.5152/eurjrheum.2015.15104] [PMID] [PMCID]

[11] Anbarian M, Mokhtari M, Zerai P, Yalfani A. [A comparison of postura control characteristics between subjects with kyphosis and controls (Persian)]. Avicenna Journal of Clinical Medicine. 2010; 16(4):53-60.

[12] McDaniels-Davidson C, Davis A, Wing D, Macera C, Lindsay S, Schousboe J, et al. Kyphosis and incident falls among community-dwelling older adults. Osteoporosis International. 2018; 29(1):163-9. [DOI:10.1007/ s00198-017-4253-3] [PMID]

[13] Yagi M, Kaneko S, Yato Y, Asazuma T. Standing balance and compensatory mechanisms in patients with adult spinal deformity. Spine. 2017; 42(10):E584-E91. [DOI:10.1097/BRS.0000000000001901] [PMID]

[14] Sedaghati P. [Applied kinanthropometry (Persian)]. Guilan: University of Guilan; 2017.

[15] Pearsall DJ, Reid JG, Hedden DM. Comparison of three noninvasive methods for measuring scoliosis. Physical Therapy. 1992; 72(9):648-57. [DOI:10.1093/ptj/72.9.648] [PMID]

[16] Hart DL, Rose SJ. Reliability of a noninvasive method for measuring the lumbar curve. Journal of Orthopedic \& Sports Physical Therapy. 1986; 8(4):180-4. [DOI:10.2519/jospt.1986.8.4.180] [PMID]

[17] Thigpen CA, Padua DA, Michener LA, Guskiewicz K, Giuliani C, Keener JD, et al. Head and shoulder posture affect scapular mechanics and mus- cle activity in overhead tasks. Journal of Electromyography and Kinesiology. 2010; 20(4):701-9. [DOI:10.1016/j.jelekin.2009.12.003] [PMID]

[18] Harman K, Hubley-Kozey CL, Butler H. Effectiveness of an exercise program to improve forward head posture in normal adults: A randomized, controlled 10-week trial. Journal of Manual \& Manipulative Therapy. 2005; 13(3):163-76. [DOI:10.1179/106698105790824888]

[19] Taheri M, Irandoust K, Norasteh AA, Shaviklo J. [The effect of combined core stability and neuromuscular training on postureal control in students with congenital hearing loss (Persian)]. Journal of Research in Rehabilitation Sciences. 2017; 13(2):80-6

[20] Cioni M, Cocilovo A, Rossi F, Paci D, Valle MS. Analysis of ankle kinetics during walking in individuals with Down syndrome. American Journal on Mental Retardation. 2001; 106(5):470-8. [DOI:10.1352/08958017(2001)1062.0.CO;2]

[21] Seyedi M, Seydi F, Rahimi A, Minoonejad H. [An investigation of the ef ficiency of sensory systems involved in postural control in deaf athletes and non-athletes (Persian)]. Journal of Sport Medicine. 2015; 7(1):11127. [DOI:10.13140/RG.2.1.4785.0328]

[22] Akbari A, Ghiasi F, Papoli R, Jalali MA. [A relationship between static and dynamic postural stability index and anthropometrics index in healthy men and women with normal BMI index (Persian)]. Journal of Sabzevar University of Medical Science. 2014; 21(2):241-51. 
This Page Intentionally Left Blank 